\title{
Electrical Stimulation Over Human Posterior Parietal Cortex Selectively Enhances the Capacity of Visual Short-Term Memory
}

\author{
(DSisi Wang, ${ }^{1,2 \star}$ (irawaj Itthipuripat, ${ }^{2,3,4 *}$ and $\odot Y$ Yixuan $\mathrm{Ku}^{1,5}$ \\ ${ }^{1}$ Shanghai Key Laboratory of Brain Functional Genomics, Shanghai Changning-ECNU Mental Health Center, School of Psychology and Cognitive Science, \\ East China Normal University, Shanghai 200062, People's Republic of China, 2Department of Psychology, Center for Integrative and Cognitive \\ Neuroscience, and Interdisciplinary Program in Neuroscience, Vanderbilt University, Nashville, Tennessee 37235, ${ }^{3}$ Learning Institute, and 4 Futuristic \\ Research in Enigmatic Aesthetics Knowledge Laboratory, King Mongkut's University of Technology Thonburi, Bangkok 10140, Thailand, and ${ }^{5} \mathrm{NYU}$-ECNU \\ Institute of Brain and Cognitive Science, NYU Shanghai and Collaborative Innovation Center for Brain Science, Shanghai 200062, People's Republic of China
}

Visual short-term memory (VSTM) provides an on-line mental space for incoming sensory information to be temporally maintained to carry out complex behavioral tasks. Despite its essential functions, the capacity at which VSTM could maintain sensory information is limited (i.e., VSTM can hold only about three to four visual items at once). Moreover, the quality of sensory representation (i.e., precision) degrades as more information has to be maintained in VSTM. Correlational evidence suggests that the level and the pattern of neural activity measured in the posterior parietal cortex (PPC) track both VSTM capacity and precision. However, the causal contributions of the PPC to these different VSTM operations are unclear. Here, we tested whether stimulating the PPC with transcranial direct current stimulation (tDCS) could increase VSTM capacity or precision. We found that stimulating the PPC in male and female human participants selectively enhanced VSTM capacity when the number of memory items exceeded capacity limit, without significant effects on VSTM precision. Moreover, this enhancement of VSTM capacity is region specific as stimulating the prefrontal cortex did not change VSTM capacity or precision. Null stimulation effects in the sensory memory condition confirmed that the tDCS-induced enhancement of VSTM capacity was not simply due to changes in sensory or attentional processes. Altogether, these results provide causal evidence suggesting that the PPC has a more dominant role in supporting the storage capacity of VSTM compared with maintaining the quality of sensory representations. Furthermore, tDCS could be used as a promising noninvasive method to enhance this PPC VSTM-related function.

Key words: capacity; posterior parietal cortex; precision; tDCS; visual short-term memory

Significance Statement

Correlational evidence from neuroimaging and electrophysiology suggests that the posterior parietal cortex (PPC) supports the storage capacity of visual short-term memory (VSTM) and the precision of sensory representations maintained in VSTM. However, the causal contributions of the PPC to these different VSTM functions were unclear. Here, we found that electrical stimulation over the PPC selectively enhanced VSTM capacity without changing VSTM precision. Overall, our findings suggest that the PPC has a dominant and causal role in supporting the storage capacity of VSTM.

\section{Introduction}

Visual short-term memory (VSTM) provides an on-line workspace for sensory information to be maintained and manipulated

Received July 31, 2018; revised Oct. 31, 2018; accepted Nov. 9, 2018.

Author contributions: S.W. and Y.K. designed research; S.W. performed research; S.W. and S.I. analyzed data; S.W., S.I., and Y.K. wrote the paper.

This work was supported by the National Social Science Foundation (Grant 17ZDA323), the Major Program of Science and Technology Commission Shanghai Municipal (Grant 17JC1404100), Shanghai Pujiang Talents Plan Project (Grant 16PJC022) to Y.K., and the China Scholarship Council Scholarship (201706140083) to S.W. We thank Geoffrey F. Woodman for his useful discussion.

*S.W. and S.I. contributed equally to this work.

The authors declare no competing financial interests.

Correspondence should be addressed to Yixuan Ku, School of Psychology and Cognitive Science, East China Normal University,

3663 North Zhongshan Road, Shanghai200062, People's Republic of China, E-mail:yxku@psy.ecnu.edu.cn. so that humans could carry out complex behavioral tasks at hand (Baddeley and Hitch, 1974; Cowan, 2001). Despite its essential functions, VSTM has a severe limit in the storage capacity. For example, research has demonstrated that humans can only remember up to three to four visual items in mind at once (Sperling, 1960; Luck and Vogel, 1997; Cowan, 2001). Importantly, the storage capacity of VSTM among individuals is highly correlated with high-order cognitive abilities such as IQ (Conway et al., 2002; Colom et al., 2008) and academic performance (Swanson, 1994; Alloway and Alloway, 2010). Moreover, VSTM-related 
functions decline with age (Gazzaley et al., 2005) and are impaired in individuals with neuropsychiatric disorders, such as schizophrenia and major depression (Lee and Park, 2005; Rose and Ebmeier, 2006). Thus, finding effective ways to improve VSTM function is potentially a promising way to enhance learning abilities and cognitive functions across healthy individuals and clinical populations.

For the past few decades, there has been converging correlational evidence suggesting that the posterior parietal cortex (PPC) supports the maintenance of sensory information in VSTM. Seminal studies using univariate analyses of functional magnetic resonance imaging (fMRI) and electroencephalography (EEG) data have found that the level of PPC activity tracks the number of items maintained in VSTM and reaches an asymptotic level until VSTM is filled (at approximately three to four items), which is known as the limited capacity level of VTSM (Todd and Marois, 2004; Vogel and Machizawa, 2004; Xu and Chun, 2006). Importantly, the levels of PPC activity at these set sizes precisely predict individual differences in VSTM capacity, suggesting a tight relationship between the PPC activity and the storage capacity of VSTM (Vogel and Machizawa, 2004; Fukuda et al., 2015a,b). Another line of research has also suggested that the PPC plays a role in preserving the quality of sensory representations in VSTM (i.e., VSTM precision). Using multivariate analyses of EEG and fMRI data, recent studies have shown that PPC encodes content-specific sensory representations maintained in VSTM (Sprague et al., 2014, 2016; Ester et al., 2015; Bettencourt and Xu, 2016; Galeano Weber et al., 2016, 2017; Yu and Shim, 2017), and the quality of mnemonic contents in the pattern of PPC activity correlates with the variability of response times and VSTM precision, obtained behaviorally, across trials and individual subjects (Bettencourt and Xu, 2016; Galeano Weber et al., 2016).

Together, converging correlational evidence suggests that the PPC is involved in supporting both VSTM capacity and precision. However, its causal contributions to these different VSTM functions are unclear. To investigate this, we applied anodal transcranial direct current stimulation (tDCS) over the PPC of human participants before they performed a variant of VSTM task where they recalled the orientations of memorized visual items varying in set sizes (two, four, or six items). We simultaneously measured VSTM capacity and precision by modeling the response error distribution (Zhang and Luck, 2008, 2011). To control for sensory and attentional confounds induced by PPC stimulation, subjects also encountered sensory memory (SM) trials where the delay period was much shorter than that in VSTM trials (100 ms in SM vs 1000 ms in VSTM). Across different days, subjects also received sham stimulation and anodal tDCS over the dorsolateral prefrontal cortex (DLPFC) to control for placebo and general excitability effects, respectively. We found that anodal PPC tDCS selectively enhanced VSTM capacity (relative to sham and DLPFC stimulation) when it surpassed the capacity limit (i.e., set size 6) without changing VSTM precision. There was no stimulation effect in SM trials, confirming that the PPC stimulation effect was specific to VSTM function, and not to changes in sensory or attentional processes. Our results, therefore, suggest that the PPC has a dominant and causal role in supporting the storage capacity of VSTM.

\section{Materials and Methods}

Participants. We recruited 20 male and female human adults from the East China Normal University (ECNU) to participate in the study (14 females, all right handed; mean age, $22.9 \pm 1.94$ years). All participants had normal or corrected-to-normal vision, no metallic implant, and no history of any neurological or psychiatric illness. Before their participa- tion, all subjects provided written informed consent, as required by the ethics committee at ECNU. The recruitment of 20 subjects is within the typical range of tDCS studies using similar within-subject designs and multisession approaches (10-20 subjects in Tseng et al., 2012; Reinhart and Woodman, 2014, 2015; Heinen et al., 2016; Reinhart et al., 2016).

Stimuli and tasks. Stimuli were controlled by a 12.5 inch laptop running MATLAB (R2011b; MathWorks) and the Psychophysics Toolbox (version 3.0.12; Brainard, 1997; Pelli, 1997). Subjects were seated $60 \mathrm{~cm}$ from the monitor with a gray background of $80 \mathrm{~cd} / \mathrm{m}^{2}$ (refresh rate, 60 $\mathrm{Hz}$; resolution, $1024 \times 768 ; \mathrm{R} / \mathrm{G} / \mathrm{B}, 192 / 192 / 192)$. The experiment was performed in a noise-isolated room. Figure $1 a$ depicts a schematic of the experimental paradigm. Each trial started with a stimulus display containing two, four, or six different oriented black bars (visual angle, $2^{\circ} \times$ $0.3^{\circ}$; luminance, $\left.1 \mathrm{~cd} / \mathrm{m}^{2} ; \mathrm{R} / \mathrm{G} / \mathrm{B}, 0 / 0 / 0\right)$. Individual bars were presented at an eccentricity of $6^{\circ}$ visual angle and were at least a $2^{\circ}$ visual angle apart from one another. Half of the stimuli was presented on the left, and the other half was presented on the right hemifield. The orientations of these stimuli were randomly chosen from $10^{\circ}$ to $170^{\circ}$ and were at least $10^{\circ}$ apart from one another. The stimulus array was presented for $200 \mathrm{~ms}$ followed by a blank delay for $100 \mathrm{~ms}$ for SM trials or $1000 \mathrm{~ms}$ for VSTM trials. After the blank screen, a black circular probe $\left(2^{\circ}\right.$ visual angle inner diameters; $0.3^{\circ}$ visual angle thickness) appeared at one of the previously presented stimulus locations. Participants were instructed to report the orientation of the remembered stimulus at the probed location by clicking on the black circular probe using a mouse as precisely as possible (on either side of the bar to produce a virtual bar across the center of the circle). The location of the probed target was pseudorandomly selected. Intertrial intervals were jittered from 1000 to $2000 \mathrm{~ms}$.

On the first day, participants completed a practice session with the VSTM recall task with set size 2 for 50 trials. Then, they received 15 min of PPC, DLPFC, or sham stimulation. Immediately after stimulation ended, they completed three blocks of SM and three blocks of VSTM. Set size was manipulated on a block-by-block basis. Each block consisted of 60 trials. Block order was pseudorandomized within each session. Half of the subjects started with the SM task, and the other half started with the VSTM task.

Data analysis. First, we obtained the recall error distribution by calculating the angular distance between the actual orientation and the reported orientation for each trial. Next, the data were sorted into different set sizes, memory types, and stimulation protocols. Then, we fit each response error distribution with the standard mixture model (Zhang and Luck, 2008) using a maximum likelihood estimation procedure implemented in the MemToolbox in MATLAB (Suchow and Brady, 2013) to obtain capacity and precision parameters. According to the standard mixture model, response deviations from the actual orientation reflect a mixture of trials where the probed bars were remembered and trials where observers guessed randomly (Fig. 1b). Therefore, the distribution of recall errors consists of a mixture of a von Mises distribution [the remembered representation: similar to circular Gaussian distribution around the correct orientation (SD)] and a uniform distribution [random guesses $(g)$ ]. Parameter SD is the SD of the von Mises distribution, which represents the width of the recall error distribution of trials that the probed item was remembered. Memory precision is an inverse of SD $\left(\mathrm{SD}^{-1}\right)$. Parameter $g$ is the height of the uniform distribution representing the guessing probability, which can be used to calculate the probability at which the probed item was remembered in memory $(P m=1-g)$. Memory capacity $(K)$ was then obtained from multiplying $P m$ with set size. Note that we chose the standard mixture model over the swap model (Bays and Husain, 2008) because the Akaike information criterion (AIC) indicated better fits for the standard mixture over the swap models in 18 of 20 subjects and in $85 \%$ of all experimental conditions. Moreover, the Bayesian information criterion (BIC) indicated that the standard mixture model provided better fits for every subject and in $92 \%$ of all conditions. The AIC and BIC values of each participant in all conditions are listed in Tables 1 and 2.

tDCS setup. tDCS was delivered by a battery-driven constant current stimulator (Eldith, NeuroCon) using a pair of rubber electrodes in a $5 \times$ $7 \mathrm{~cm}^{2}$ saline-soaked synthetic sponge. Individual subjects underwent PPC stimulation, DLPFC stimulation, and sham stimulation on 3 differ- 
a

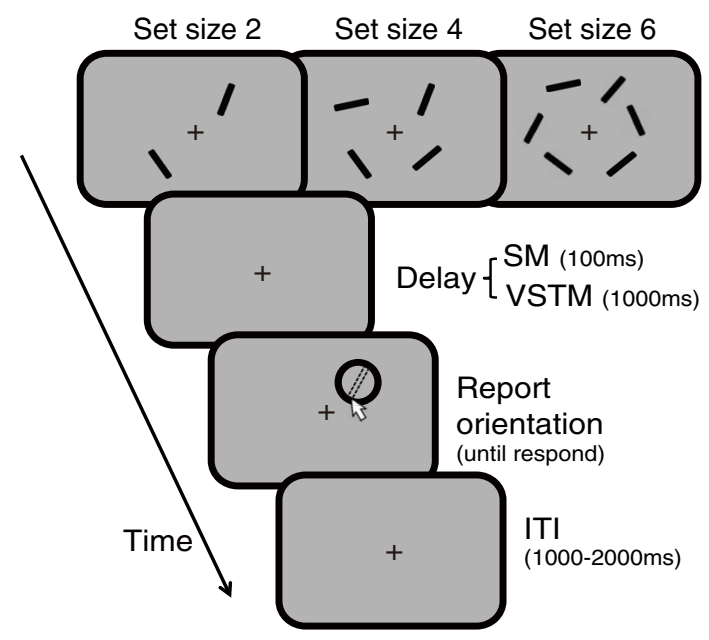

b

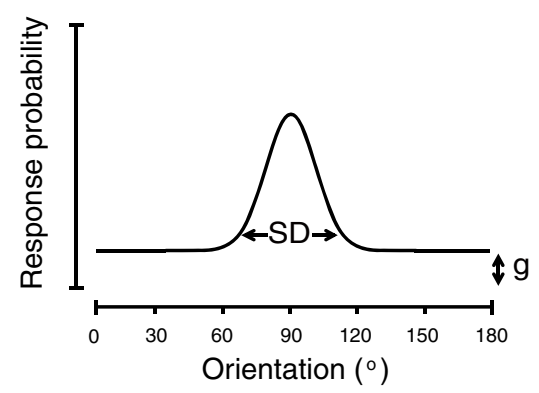

C

Stimulus samples (200ms)

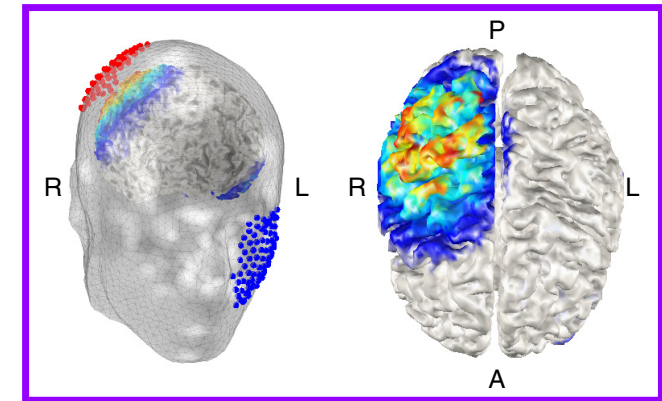

$\mathrm{J}\left(\mathrm{Am}^{-2}\right)$
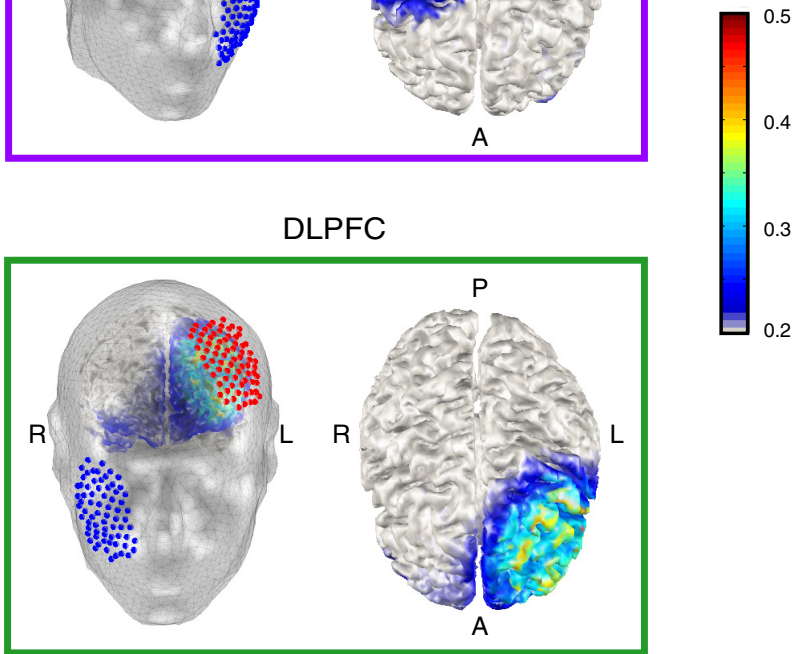

Figure 1. Experimental procedure. $a$, Schematic diagrams of the SM and VSTM continuous recall task. The delay intervals of SM and VSTM trials are 100 and $1000 \mathrm{~ms}$, respectively. The unfilled bar in the probe circle will not appear in the real task, it is an illustration. $\boldsymbol{b}$, Standard mixture model of performance. The solid black line comprises a mixture of two kinds of trials: trials where items are remembered in memory and trials where items are not remembered in memory. Parameter $g$ represents the height of the uniform distribution, and SD illustrates the width of recall error distribution in memory. C, Current density distributions for anodal PPC tDCS (top) and anodal DLPFC DCS (bottom). The left panels show electrode locations on the scalp surface and the contralateral cheek as well as cortical current density distributions from a front view. The right panels show cortical current density distributions from an overhead view. L, Left; R, right; A, anterior; P, posterior; ITI, intertrial interval.

Table 1. The difference in AIC values obtained from the standard mixture model and the swap model, the lower AIC values represent better model fit

\begin{tabular}{|c|c|c|c|c|c|c|c|c|c|c|c|c|c|c|c|c|c|c|c|c|}
\hline AIC (SMM-SWM) & par01 & par02 & par03 & par04 & par05 & par06 & par07 & par08 & par09 & par10 & par11 & par12 & par13 & par14 & par15 & par16 & par17 & par18 & par19 & par20 \\
\hline \multicolumn{21}{|l|}{ WM } \\
\hline \multicolumn{21}{|l|}{ Sham } \\
\hline Set size 2 & -2.00 & -2.05 & -2.00 & -2.00 & -2.00 & -2.00 & -2.00 & -2.00 & -2.16 & -2.00 & -2.00 & -2.00 & -2.04 & -2.23 & -2.00 & -2.00 & -2.00 & -2.00 & -2.00 & -2.00 \\
\hline Set size 4 & -2.05 & 0.17 & -0.49 & -1.36 & -1.71 & 0.27 & -1.68 & 7.33 & 1.67 & 4.95 & -2.00 & -2.00 & -1.36 & -2.00 & -2.00 & 4.02 & -2.07 & 0.04 & -1.29 & 0.62 \\
\hline Set size6 & 2.79 & -2.00 & 0.70 & -2.00 & -2.00 & -2.00 & -2.22 & -2.00 & -0.97 & -2.00 & -2.00 & -2.00 & -2.07 & -2.06 & -2.00 & -2.00 & -1.92 & -1.84 & -1.49 & -0.14 \\
\hline \multicolumn{21}{|l|}{ PPC } \\
\hline Set size2 & -3.94 & -2.04 & -2.00 & -2.00 & -2.00 & -2.00 & -2.00 & -2.00 & -2.00 & -2.00 & -2.00 & -2.00 & -2.00 & -2.00 & -2.00 & -2.00 & -2.00 & -2.00 & -2.00 & -2.00 \\
\hline Set size4 & -2.31 & -0.02 & -2.00 & -2.00 & -2.02 & -2.00 & 3.73 & 1.26 & -1.23 & -1.70 & -2.00 & -2.00 & 1.38 & -1.53 & -0.73 & 12.21 & -2.00 & -0.55 & -2.11 & -2.00 \\
\hline Set size6 & -2.00 & 3.86 & -1.96 & -2.02 & 4.46 & -2.00 & 6.30 & -2.00 & -2.02 & -2.16 & -1.82 & -2.00 & -2.00 & -2.00 & -2.12 & -2.00 & -2.00 & -1.99 & 0.41 & -2.00 \\
\hline \multicolumn{21}{|l|}{ DLPFC } \\
\hline Set size2 & -2.00 & -2.04 & -2.01 & -2.00 & -2.00 & -2.00 & -2.00 & -2.00 & -2.00 & -2.00 & -2.00 & -2.03 & -2.00 & -2.00 & -2.00 & -2.01 & -2.10 & -2.03 & -5.82 & -2.00 \\
\hline Set size4 & -2.00 & -2.00 & 1.59 & -1.24 & 1.40 & -1.94 & 0.50 & -0.16 & -2.00 & -2.00 & 6.60 & -1.65 & -2.00 & -2.00 & -1.77 & -1.83 & -2.10 & -2.00 & -1.24 & 8.46 \\
\hline Set size6 & -2.00 & -1.12 & -1.92 & -2.05 & -2.00 & -1.02 & -2.02 & -2.02 & -1.98 & -2.00 & -2.00 & -1.62 & -1.86 & -0.53 & -2.00 & -2.00 & -2.00 & -0.43 & -1.89 & -1.03 \\
\hline \multicolumn{21}{|l|}{$S M$} \\
\hline \multicolumn{21}{|l|}{ Sham } \\
\hline Set size2 & -2.06 & -2.00 & -2.00 & -2.03 & -2.02 & -2.00 & -2.00 & -2.00 & -2.01 & -2.00 & -2.00 & -2.07 & -2.00 & -2.00 & -2.43 & -2.00 & -2.00 & -2.00 & -2.00 & -2.00 \\
\hline Set size4 & -1.10 & 11.11 & 5.19 & -0.99 & -2.00 & -2.02 & 0.62 & -0.77 & -2.00 & 5.79 & 0.80 & 8.77 & 2.16 & -1.18 & -2.00 & -2.00 & -2.01 & -2.00 & -1.84 & -2.00 \\
\hline Set size6 & -2.00 & -5.37 & -1.95 & -2.00 & -2.00 & 0.34 & 2.97 & -1.56 & -2.00 & -2.03 & -2.94 & -1.36 & -2.00 & -1.78 & -2.00 & -0.61 & -2.00 & -2.00 & -2.00 & -2.00 \\
\hline \multicolumn{21}{|l|}{ PPC } \\
\hline Set size2 & -2.00 & -2.00 & -2.00 & -2.00 & -2.00 & -2.00 & -2.00 & -2.00 & -0.62 & -2.09 & -2.00 & -2.00 & -2.00 & -2.05 & -2.24 & -2.00 & -2.00 & -2.00 & -2.37 & -2.00 \\
\hline Set size4 & -2.00 & -0.27 & -2.00 & 3.55 & -0.65 & -2.00 & 0.78 & 8.67 & -2.00 & -2.00 & -1.10 & 7.34 & -1.65 & -2.00 & -2.00 & -2.00 & -2.00 & -1.31 & -2.00 & 1.16 \\
\hline Set size6 & -1.96 & -2.00 & -2.00 & -2.01 & -2.00 & -2.00 & 2.38 & 1.74 & -2.00 & -0.55 & -2.00 & -2.00 & -1.47 & -2.04 & -1.67 & -2.00 & -2.00 & -2.00 & 0.90 & -0.52 \\
\hline \multicolumn{21}{|l|}{ DLPFC } \\
\hline Set size2 & -2.00 & -2.35 & -2.01 & -2.00 & -2.00 & -2.00 & -2.00 & -2.00 & -2.00 & -2.03 & -2.00 & -2.00 & -2.00 & -2.00 & -2.00 & -2.00 & -2.00 & -2.00 & -2.00 & -2.00 \\
\hline Set size4 & -1.74 & 20.28 & -1.35 & -2.00 & -2.00 & -2.01 & 2.23 & -1.97 & -2.00 & 6.44 & -2.00 & -0.76 & -1.03 & 0.09 & 6.34 & -1.96 & -2.00 & -1.08 & 1.19 & 17.13 \\
\hline Set size6 & -2.00 & 4.26 & -2.00 & -2.00 & -1.77 & -1.81 & -2.00 & -2.00 & -0.45 & 4.56 & -2.01 & 0.12 & -2.00 & -2.00 & -2.00 & -1.73 & -1.93 & 6.62 & -1.79 & 0.02 \\
\hline
\end{tabular}

SMM, Standard mixture model; SWM, swap model. 
Table 2. The difference in BIC values obtained from the standard mixture model and the swap model, the lower BIC values represent better model fit

\begin{tabular}{|c|c|c|c|c|c|c|c|c|c|c|c|c|c|c|c|c|c|c|c|c|}
\hline BIC (SMM-SWM) & par01 & par02 & par03 & par04 & par05 & par06 & par07 & par08 & par09 & par10 & par11 & par12 & par13 & par14 & par15 & par16 & par17 & par18 & par19 & par20 \\
\hline \multicolumn{21}{|l|}{ WM } \\
\hline \multicolumn{21}{|l|}{ Sham } \\
\hline Set size 2 & -4.09 & -4.14 & -4.09 & -4.09 & -4.09 & -4.10 & -4.09 & -4.09 & -4.26 & -4.09 & -4.09 & -4.09 & -4.13 & -4.32 & -4.09 & -4.09 & -4.09 & -4.09 & -4.09 & -4.09 \\
\hline Set size 4 & -4.14 & -1.92 & -2.58 & -3.45 & -3.80 & -1.83 & -3.78 & 5.23 & -0.42 & 2.86 & -4.09 & -4.09 & -3.46 & -4.10 & -4.09 & 1.93 & -4.17 & -2.05 & -3.39 & -1.48 \\
\hline Set size 6 & 0.70 & -4.09 & -1.40 & -4.10 & -4.09 & -4.10 & -4.31 & -4.10 & -3.07 & -4.09 & -4.09 & -4.09 & -4.16 & -4.15 & -4.09 & -4.09 & -4.01 & -3.94 & -3.58 & -2.23 \\
\hline \multicolumn{21}{|l|}{ PPC } \\
\hline Set size 2 & -6.03 & -4.14 & -4.09 & -4.09 & -4.09 & -4.09 & -4.09 & -4.09 & -4.09 & -4.09 & -4.09 & -4.09 & -4.10 & -4.09 & -4.10 & -4.09 & -4.09 & -4.09 & -4.09 & -4.09 \\
\hline Set size 4 & -4.41 & -2.11 & -4.09 & -4.09 & -4.11 & -4.09 & 1.64 & -0.83 & -3.33 & -3.79 & -4.10 & -4.10 & -0.71 & -3.62 & -2.83 & 10.12 & -4.09 & -2.65 & -4.20 & -4.10 \\
\hline Set size 6 & -4.09 & 1.77 & -4.05 & -4.11 & 2.37 & -4.09 & 4.20 & -4.09 & -4.12 & -4.26 & -3.91 & -4.09 & -4.10 & -4.09 & -4.22 & -4.09 & -4.10 & -4.08 & -1.68 & -4.09 \\
\hline \multicolumn{21}{|l|}{ DLPFC } \\
\hline Set size 2 & -4.09 & -4.13 & -4.10 & -4.09 & -4.09 & -4.09 & -4.09 & -4.09 & -4.09 & -4.09 & -4.09 & -4.12 & -4.09 & -4.09 & -4.09 & -4.10 & -4.20 & -4.12 & -7.92 & -4.09 \\
\hline Set size 4 & -4.10 & -4.09 & -0.50 & -3.34 & -0.70 & -4.04 & -1.59 & -2.26 & -4.09 & -4.09 & 4.50 & -3.75 & -4.09 & -4.09 & -3.87 & -3.93 & -4.19 & -4.09 & -3.33 & 6.37 \\
\hline Set size 6 & -4.09 & -3.21 & -4.02 & -4.14 & -4.09 & -3.11 & -4.12 & -4.12 & -4.08 & -4.09 & -4.09 & -3.71 & -3.96 & -2.62 & -4.09 & -4.09 & -4.10 & -2.52 & -3.99 & -3.12 \\
\hline \multicolumn{21}{|l|}{ SM } \\
\hline \multicolumn{21}{|l|}{ Sham } \\
\hline Set size 2 & -4.15 & -4.10 & -4.09 & -4.12 & -4.11 & -4.09 & -4.09 & -4.09 & -4.11 & -4.09 & -4.09 & -4.16 & -4.09 & -4.09 & -4.52 & -4.09 & -4.09 & -4.09 & -4.09 & -4.09 \\
\hline Set size 4 & -3.20 & 9.02 & 3.10 & -3.08 & -4.09 & -4.11 & -1.48 & -2.87 & -4.10 & 3.70 & -1.30 & 6.67 & 0.07 & -3.27 & -4.09 & -4.10 & -4.10 & -4.09 & -3.93 & -4.09 \\
\hline Set size 6 & -4.09 & -7.46 & -4.05 & -4.10 & -4.09 & -1.75 & 0.88 & -3.65 & -4.10 & -4.13 & -5.03 & -3.45 & -4.09 & -3.88 & -4.09 & -2.71 & -4.09 & -4.09 & -4.09 & -4.09 \\
\hline \multicolumn{21}{|l|}{ PPC } \\
\hline Set size 2 & -4.09 & -4.09 & -4.09 & -4.09 & -4.10 & -4.09 & -4.09 & -4.09 & -2.72 & -4.19 & -4.09 & -4.10 & -4.09 & -4.14 & -4.33 & -4.09 & -4.09 & -4.10 & -4.47 & -4.09 \\
\hline Set size 4 & -4.09 & -2.36 & -4.09 & 1.46 & -2.74 & -4.09 & -1.31 & 6.57 & -4.10 & -4.09 & -3.20 & 5.24 & -3.74 & -4.09 & -4.09 & -4.10 & -4.09 & -3.40 & -4.09 & -0.93 \\
\hline Set size 6 & -4.05 & -4.09 & -4.09 & -4.10 & -4.09 & -4.09 & 0.28 & -0.35 & -4.09 & -2.64 & -4.09 & -4.09 & -3.57 & -4.13 & -3.77 & -4.09 & -4.09 & -4.09 & -1.20 & -2.61 \\
\hline \multicolumn{21}{|l|}{ DLPFC } \\
\hline Set size 2 & -4.09 & -4.44 & -4.10 & -4.09 & -4.09 & -4.09 & -4.10 & -4.09 & -4.10 & -4.12 & -4.09 & -4.10 & -4.09 & -4.09 & -4.09 & -4.09 & -4.09 & -4.09 & -4.09 & -4.09 \\
\hline Set size 4 & -3.84 & 18.19 & -3.44 & -4.09 & -4.09 & -4.10 & 0.14 & -4.07 & -4.10 & 4.35 & -4.09 & -2.85 & -3.13 & -2.00 & 4.24 & -4.06 & -4.09 & -3.18 & -0.90 & 15.04 \\
\hline Set size 6 & -4.10 & 2.16 & -4.09 & -4.10 & -3.87 & -3.91 & -4.10 & -4.09 & -2.54 & 2.47 & -4.11 & -1.97 & -4.10 & -4.09 & -4.09 & -3.83 & -4.03 & 4.52 & -3.88 & -2.08 \\
\hline
\end{tabular}

SMM, Standard mixture model; SWM, swap model.

ent days, separated by at least $48 \mathrm{~h}$ to minimize potential carryover effects related to repeated exposure to brain stimulation. The order of stimulation types was counterbalanced across participants. For PPC stimulation, the anodal electrode was placed over P4 according to the International 10-20 system for EEG electrode placement, and the reference electrode was placed over the left cheek (Tseng et al., 2012; Xue et al., 2012; Hsu et al., 2014; Li et al., 2017). Here, we took the right hemisphere as our stimulation site because existing studies have found that the right hemisphere is more dominant in VSTM than the left hemisphere (Vogel et al., 2003; Owen et al., 2005; Habekost and Rostrup, 2007; van der Ham et al., 2009; Fukuda et al., 2015a). For DLPFC stimulation, the anodal electrode was placed over $\mathrm{F} 3$ and the reference electrode was placed over the right cheek (Fregni et al., 2005; Ohn et al., 2008; Elmer et al., 2009; Jo et al., 2009; Zaehle et al., 2011). We used DLPFC as a control site to exclude potential confounds from general excitability changes from tDCS. During PPC and DLPFC stimulation, a constant current of $2.0 \mathrm{~mA}$ was applied continuously for $15 \mathrm{~min}$, with a linear fade in and fade out of $20 \mathrm{~s}$. For sham condition, the electrode placement was either the same as PPC stimulation in half of the subjects or the same as DLPFC stimulation in the other half. Unlike the actual stimulation protocol, the duration of sham stimulation lasted for $30 \mathrm{~s}$ from the initial time of the stimulation. This ensured that in the sham condition participants experienced a similar itching feeling that receded over the first few seconds of active stimulation.

Experimental design and statistical analysis. The experiment was a within-subject design of the following three factors: memory tasks (SM/ VSTM), set size conditions (2/4/6), and stimulation conditions (sham/ PPC/DLPFC). All statistical tests on the capacity and precision parameters were performed in SPSS version 19.0 (IBM). Two participants were excluded from the following analyses due to their poor performance in VSTM set size 6 ( $>2.5$ SDs from all participants). Note that the statistical results were qualitatively similar without excluding these two subjects. First, we performed two-way repeated-measures ANOVAs with within-subject factors of memory tasks (SM/VSTM) and set size conditions $(2 / 4 / 6)$ on the capacity and precision parameters. Then, we performed one-way repeated-measures ANOVAs with a within-subject factor of set size separately for the SM and VSTM tasks. Post hoc paired $t$ tests were then performed to test parameter differences between set sizes 2 and 4, 2 and 6, and 4 and 6 for each memory task, and multiple comparisons were corrected using the Bonferroni method.
To examine the effects of PPC and DLPFC stimulation on the capacity and precision parameters, we first subtracted the parameters from the sham condition of those obtained from the active stimulation (PPC and DLPFC stimulation) separately for each set size and memory task. Then, we used paired $t$ tests to compare these normalized values against zeroes, and multiple comparisons were corrected using the Bonferroni method (the threshold was set as 0.0083 , which was the quotient of 0.05 divided by 6). To test the main effects of stimulated regions and set size as well as their interaction on these normalized parameters, we used two-way repeated-measures ANOVAs with within-subject factors of stimulated regions (PPC/DLPFC) and set size (2/4/6). Since we found a selective enhancement of the VSTM capacity parameter at set size 6 with PPC stimulation, a followed-up pairwise $t$ test was performed to officially examine whether this enhancement was statistically higher than the effect of DLPFC stimulation at this set size. Another followed-up $t$ test was also performed to test whether this capacity enhancement was higher than the normalized capacity value in the SM task. The effect sizes for all ANOVAs and $t$ test results were also reported as $\eta_{p}^{2}$ and Cohen's $d$, respectively.

In addition, we also estimated the statistical power with the sample size of current study using $G^{\star}$ Power analysis (Faul et al., 2009). With a sample size of 18 and an effect size of 1.028 for the $t$ test between VSTM capacity in set size 6 condition after sham and PPC tDCS stimulation $\left(t_{(17)}=\right.$ $4.291, p<0.001$, Cohen's $d=1.028$; see Results), if the probability of type I error $(\alpha)$ is set as 0.05 , we could reject the null hypothesis with probability (power, $1-\beta$ ) of 0.984 , which means that the statistical power of the sample size in the present study is reasonably high.

Current-flow model. To visualize the current density distributions of our tDCS protocols, we used an open-source MATLAB-based toolbox, COMETS (computation of electric field due to transcranial current stimulation; the toolbox package is freely available at http://www.cometstool. com) to simulate local electric fields. COMETS has been previously used to investigate inconsistent outcomes of tDCS that may depend on anatomical differences (Kim et al., 2014) and to model the effect of electrode displacement on tDCS (Ramaraju et al., 2018).

The head model used in this simulation was extracted from standard Montreal Neurological Institute brain atlas (Collins et al., 1994). It uses a three-layer boundary element method consisting of the scalp, skull boundaries, and CSF, as well as a cortical surface model extracted from MRI T1 images of standard brain atlas via CURRY6. Conductivity values for the scalp, skull, and CSF were set as $0.22,0.014$, and $1.79 \mathrm{~S} / \mathrm{m}$, respec- 
a

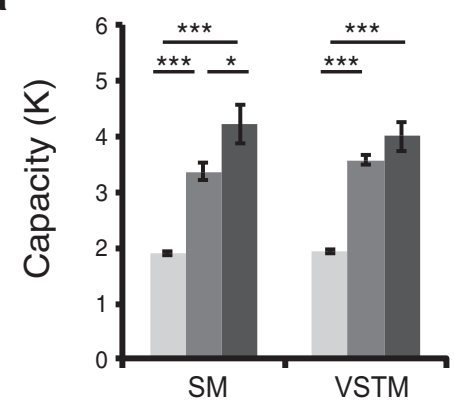

b

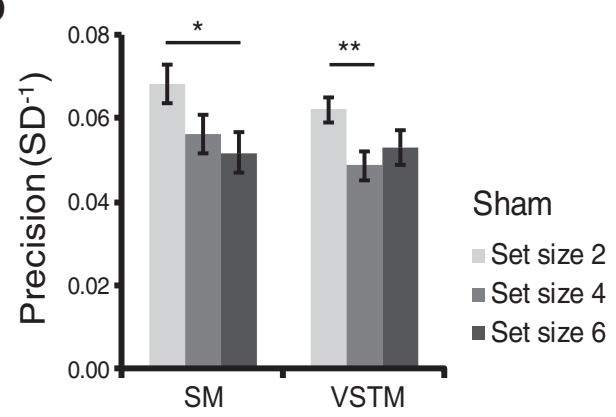

Figure 2. Set size effects on capacity and precision parameters. $\boldsymbol{a}$, Mean capacity values in the sham condition. $\boldsymbol{b}$, Mean precision values in the sham stimulation. Error bars indicate the SEM. Asterisks indicate the significant differences between set sizes with Bonferroni correction: ${ }^{*} p<0.05,{ }^{* *} p<0.01$, and ${ }^{* * *} p<0.001$.

tively (Haueisen et al., 1997). After setting the bipolar electrode configurations (position, size, and intensity) on the head model, a 3D finite element modeling based on electrostatic Laplace equation was adopted to analyze the current density inside the human head produced by tDCS (Jung et al., 2013).

\section{Results}

Set size effects on capacity and precision parameters

To examine set size effects on capacity and precision parameters across the SM and VSTM tasks, we used two-way repeatedmeasures ANOVAs with within-subject factors of set size (2/4/6) and task (SM/VSTM) on the parameters obtained from the sham condition. For the capacity parameter $(K)$, the set size effect was significant $\left(F_{(2,34)}=64.085, p<0.001, \eta_{p}^{2}=0.790\right)$. However, there were no task effects $\left(F_{(1,17)}=0.002, p=0.968, \eta_{p}^{2}=0.000\right)$ and no interactions between task and set size $\left(F_{(2,34)}=1.288, p=\right.$ $\left.0.289, \eta_{p}^{2}=0.070\right)$. Follow-up one-way repeated-measures ANOVAs revealed that there were significant set size effects in both SM $\left(F_{(2,34)}=35.982, p=0.000, \eta_{p}^{2}=0.679\right)$ and VSTM $\left(F_{(2,34)}=57.727, p=0.000, \eta_{p}^{2}=0.773\right)$. Post hoc pairwise $t$ tests showed that the significant set size effect in SM was driven by differences between set sizes 2 and $4\left(t_{(17)}=-9.111, p<0.001\right.$, Cohen's $d=3.071)$, between set sizes 2 and $6\left(t_{(17)}=-6.695, p<\right.$ 0.001 , Cohen's $d=2.271)$, and between set sizes 4 and $6\left(t_{(17)}=\right.$ -2.965, $p=0.026$, Cohen's $d=0.766$ ). All significant effects passed Bonferroni-corrected thresholds (see Materials and Methods). On the other hand, the significant set size effect in VSTM was driven only by differences between set sizes 2 and $4\left(t_{(17)}=\right.$ $-16.627, p=0.000$, Cohen's $d=5.681)$ and between set sizes 2 and $6\left(t_{(17)}=-8.303, p=0.000\right.$, Cohen's $\left.d=2.791\right)$, but without any difference between set sizes 4 and $6\left(t_{(17)}=-1.841, p=\right.$ 0.249 , Cohen's $d=0.534$; Fig. $2 a)$. All reported significant effects passed Bonferroni-corrected thresholds. The null-capacity effect between set sizes 4 and 6 in VSTM is consistent with those of several past studies, showing that VSTM capacity reaches a plateau after memory load exceeds three to four items (Luck and Vogel, 1997; Todd and Marois, 2004, 2005; Vogel and Machizawa, 2004; Xu and Chun, 2006; McCollough et al., 2007).

For the precision parameter $\left(\mathrm{SD}^{-1}\right)$, the set size effect was significant $\left(F_{(2,34)}=8.996, p=0.001, \eta_{p}^{2}=0.346\right)$. There were no task effects $\left(F_{(1,17)}=2.002, p=0.175, \eta_{p}^{2}=0.105\right)$, and no interaction between task and set size $\left(F_{(2,34)}=1.048, p=0.362\right.$, $\left.\eta_{p}^{2}=0.058\right)$. Follow-up one-way repeated-measures ANOVAs revealed that the set size effects were significant for both SM tasks $\left(F_{(2,34)}=4.920, p=0.013, \eta_{p}^{2}=0.224\right)$ and VSTM tasks $\left(F_{(2,34)}=\right.$ 5.731, $\left.p=0.007, \eta_{p}^{2}=0.252\right)$. Post hoc pairwise $t$ tests showed that the significant set size effect in the SM task was driven by differences between set sizes 2 and $6\left(t_{(17)}=2.929, p=0.028\right.$, Cohen's $d=0.820)$, without any difference between set sizes 2 and $4\left(t_{(17)}=2.139, p=0.142\right.$, Cohen's $\left.d=0.632\right)$, and between set sizes 4 and $6\left(t_{(17)}=0.920, p=1.000\right.$, Cohen's $\left.d=0.205\right)$. All significant effects passed Bonferroni-corrected thresholds. Different from SM, the significant set size effect in VSTM was driven by differences between set sizes 2 and $4\left(t_{(17)}=4.077, p=0.002\right.$, Cohen's $d=0.926)$, without any difference between set sizes 2 and $6\left(t_{(17)}=1.977, p=0.194\right.$, Cohen's $\left.d=0.573\right)$, and between set sizes 4 and $6\left(t_{(17)}=-1.041, p=0.937\right.$, Cohen's $d=0.241$; Fig. 2b).

\section{tDCS over PPC selectively enhanced VTSM capacity}

As an initial step to examine tDCS effects on memory capacity and precision, we subtracted the capacity and precision parameters in the sham condition from those in the active stimulation condition. This subtraction method yielded normalized capacity and precision measures, which were then compared against zeroes using pairwise $t$ tests for each set size condition (2/4/6) and stimulated region (PPC/DLPFC) separately for the $\mathrm{SM}$ and VSTM tasks (Fig. 3).

For SM, we did not observe any significant tDCS effect on the normalized capacity value for any set size or stimulated region ( $t$ value range, -2.151 to $0.329 ; p$ value range, $0.046-0.912$ (which did not pass the Bonferroni-corrected threshold of 0.0083 ); Cohen's $d$ range, $0.036-0.620)$. Consistent with this result, a two-way repeated-measures ANOVA showed no significant main effects of set size $\left(F_{(2,34)}=0.665, p=0.521, \eta_{p}^{2}=\right.$ $0.038)$ and stimulated regions $\left(F_{(1,17)}=1.758, p=0.202, \eta_{p}^{2}=\right.$ 0.094 ) and no interaction between the two factors on the normalized capacity value $\left(F_{(2,34)}=1.271, p=0.294, \eta_{p}^{2}=0.070\right)$. Similarly, for the normalized precision value, there was no significant tDCS effect compared to zeroes for any set size or stimulated region ( $t$ value range, -0.698 to $1.165 ; p$ value range, $0.260-$ 0.872; Cohen's $d$ range, 0.189-0.324). A two-way repeatedmeasures ANOVA also showed no significant main effects of set size $\left(F_{(2,34)}=0.398, p=0.675, \eta_{p}^{2}=0.023\right)$ and stimulated regions $\left(F_{(1,17)}=0.000, p=0.996, \eta_{p}^{2}=0.000\right)$, and no interaction between the two factors on the normalized precision value $\left(F_{(2,34)}=\right.$ 0.473, $p=0.627, \eta_{p}^{2}=0.027$ ).

Unlike the SM task, we found that tDCS over the PPC enhanced the normalized capacity value compared to zeroes in the VSTM task. However, the enhancement was observed only at set size $6\left[t_{(17)}=4.291, p<0.001\right.$ (passing the Bonferroni-corrected threshold of 0.0083), Cohen's $d=1.028$ ] but not at lower set sizes (set size 2: $t_{(17)}=0.855, p=0.405$, Cohen's $d=0.293$; set size 4: $t_{(17)}=-0.909, p=0.376$, Cohen's $d=0.221$ ). No significant tDCS effect was found with DLPFC stimulation at any set size ( $t$ value range, -1.617 to 0.503 ; $p$ value range, $0.124-0.821$; Cohen's $d$ range, $0.078-0.409)$. Consistent with these results, a two-way 
a

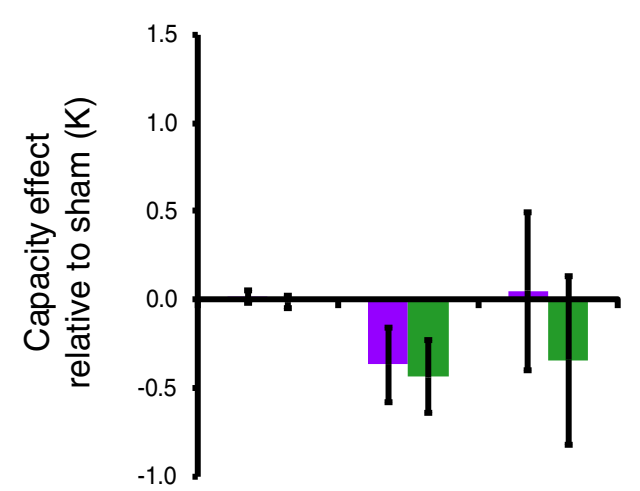

b

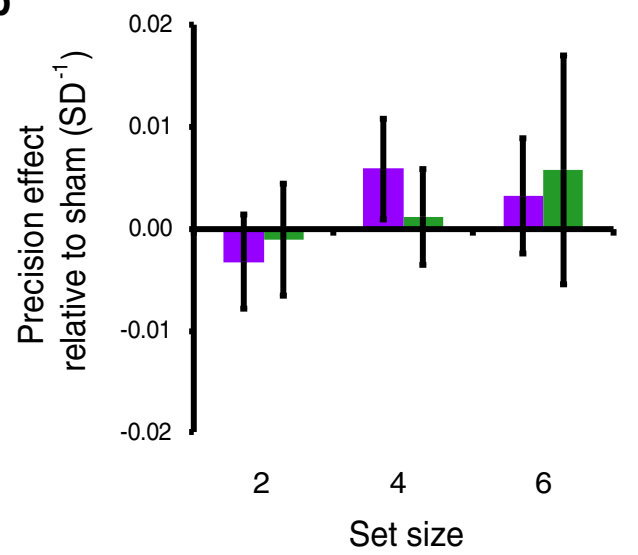

VSTM

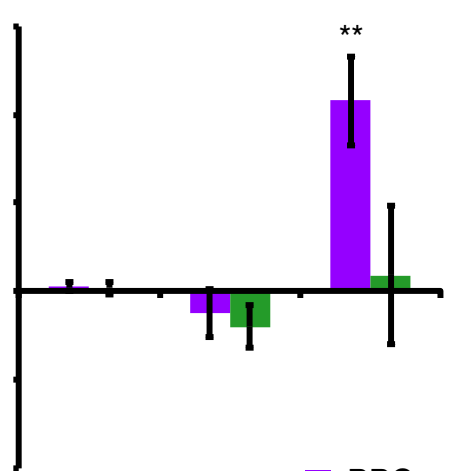

PPC

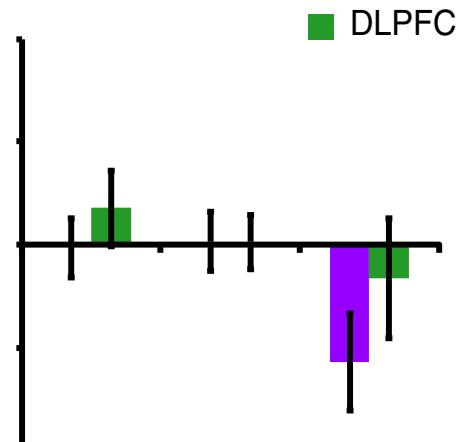

2

Figure 3. $\boldsymbol{a}, \boldsymbol{b}, \mathrm{tDCS}$ effects on the capacity $(\boldsymbol{a})$ and precision parameters $(\boldsymbol{b})$ relative to sham. Error bars indicate the SEM. ${ }^{* *} p<$ 0.01 , significant stimulation effect relative to sham with Bonferroni correction.

repeated-measures ANOVA showed significant main effects of set size $\left(F_{(2,34)}=5.002, p=0.012, \eta_{p}^{2}=0.227\right)$ and stimulated region $\left(F_{(1,17)}=6.482, p=0.021, \eta_{p}^{2}=0.276\right)$ and a significant interaction between the two factors on the normalized capacity value in the $\operatorname{VSTM}$ task $\left(F_{(2,34)}=6.089, p=0.005, \eta_{p}^{2}=0.264\right)$. Last, a followed-up pairwise $t$ test revealed that the normalized capacity value from PPC stimulation was significantly higher than the normalized capacity from DLPFC stimulation at set size $6\left(t_{(17)}=2.846, p=0.011\right.$, Cohen's $\left.d=0.711\right)$. Another followed-up $t$ test also showed that the normalized capacity value from PPC stimulation at this set size was also significantly higher in the VSTM task than that in the SM task $\left(t_{(17)}=2.231, p=\right.$ 0.039 , Cohen's $d=0.668$ ). Altogether, these follow-up comparisons confirm that the enhanced memory capacity via tDCS was specific to PPC (not DLPFC) stimulation and VSTM (not SM) function. To further explore the individual variability of tDCS effects, we showed the data of each subject in Figure 4. After the PPC stimulation, 15 of 18 subjects showed increased VSTM capacity at set size 6 and all the increments were $>13 \%$, leading to a significant enhancement effect. In contrast, the DLPFC stimulation effect was more variable across subjects. Eight subjects showed increased VSTM capacity, while the other 10 showed decreased capacity with DLPFC stimulation. Overall, these results suggest that the PPC tDCS effect on VSTM capacity was robust and highly consistent among individuals.

Unlike the capacity results, we observed no enhancement in the normalized precision parameter at any set size or with any stimulated region compared with zeroes in the VSTM task [ $t$ value range, -2.440 to $0.943 ; p$ value range, $0.026-0.919$
4

Set size (which did not pass the Bonferronicorrected threshold of 0.0083); Cohen's $d$ range, $0.015-0.628]$. The precision value at set size 6 was slightly reduced with PPC stimulation, but the effect did not pass the corrected threshold $\left(t_{(17)}=-2.440, p=\right.$ 0.026 , Cohen's $d=0.628$ ). A two-way repeated-measures ANOVA showed no significant main effect of set size $\left(F_{(2,34)}=\right.$ $\left.1.929, p=0.161, \eta_{p}^{2}=0.102\right)$, no main effect of stimulated region $\left(F_{(1,17)}=\right.$ 3.547, $\left.p=0.077, \eta_{p}^{2}=0.173\right)$, and no interaction between the two factors on the normalized precision value $\left(F_{(2,34)}=0.990\right.$, $\left.p=0.382, \eta_{p}^{2}=0.055\right)$.

\section{Discussion}

The present study tested the causal roles of the PPC in supporting VSTM capacity and precision, measured simultaneously with the VSTM recall task using the standard mixture model (Zhang and Luck, 2008). We found that anodal tDCS stimulation over the PPC selectively enhanced VSTM capacity at the highest load (set size 6) compared with sham and DLPFC stimulation, without significantly increasing VTSM precision. Overall, the results suggest that the PPC may have a relatively dominant and causal role in supporting the storage capacity of VSTM compared with its role in preserving the quality of the sensory representation hold in VSTM.

Critically, in the present study we used the SM task to control for potential sensory and attentional effects from PPC stimulation (Sparing et al., 2009; Loftus and Nicholls, 2012; Roy et al., 2015; Reinhart et al., 2016), which were not controlled for and were a major concern in previous tDCS studies (Tseng et al., 2012; Heinen et al., 2016; Li et al., 2017). Since there were no tDCS effects on the capacity and precision measurements in the SM, the PPC stimulation effect observed in the VSTM task could not be due to sensory or attentional confounds. The null effect of DLPFC stimulation also ensures that the observed capacity change with PPC stimulation is not due to global excitability changes with tDCS. Interestingly, recent studies have shown that applying anodal tDCS over the left DLPFC could enhance behavioral performance during verbal working memory (WM) tasks (Fregni et al., 2005; Ohn et al., 2008; Andrews et al., 2011). Together, the results from these studies and our present study provide causal evidence suggesting differential roles of the PPC and the DLPFC in supporting the active maintenance of visual and verbal information, respectively, as suggested by classic theories that make distinctions between the visual sketchpad and the phonological loop of working memory (Baddeley and Hitch, 1974; Smith et al., 1996; also see McCarthy et al., 1996; Wager and Smith, 2003; Walter et al., 2003).

Converging correlational evidence from fMRI and EEG studies suggests that regions within the PPC might play essential roles both in supporting the capacity storage of VSTM and in preserving the quality of sensory representations hold in VSTM (Todd and Marois, 2004; Vogel and Machizawa, 2004; Xu and Chun, 2006; Sprague et al., 2014, 2016; Ester et al., 2015; Fukuda et al., 2015a,b; Bettencourt and Xu, 2016; Galeano Weber et al., 2016, 
a

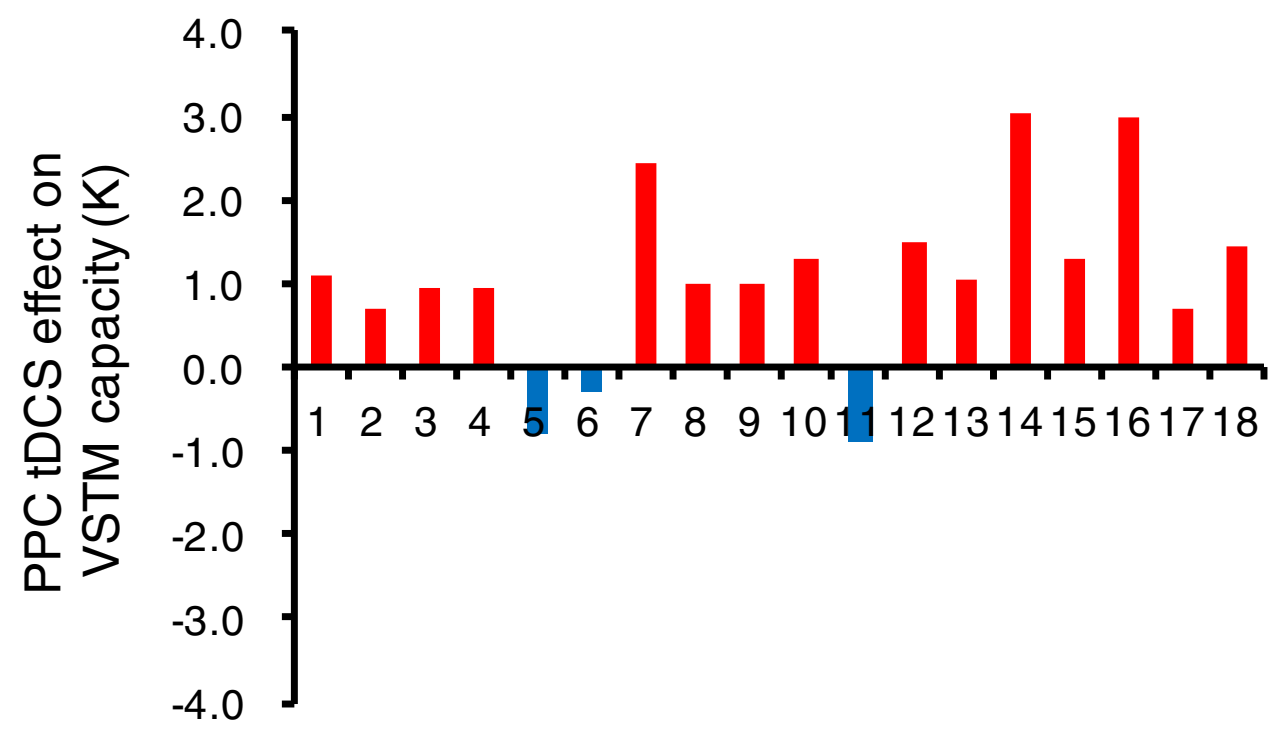

b

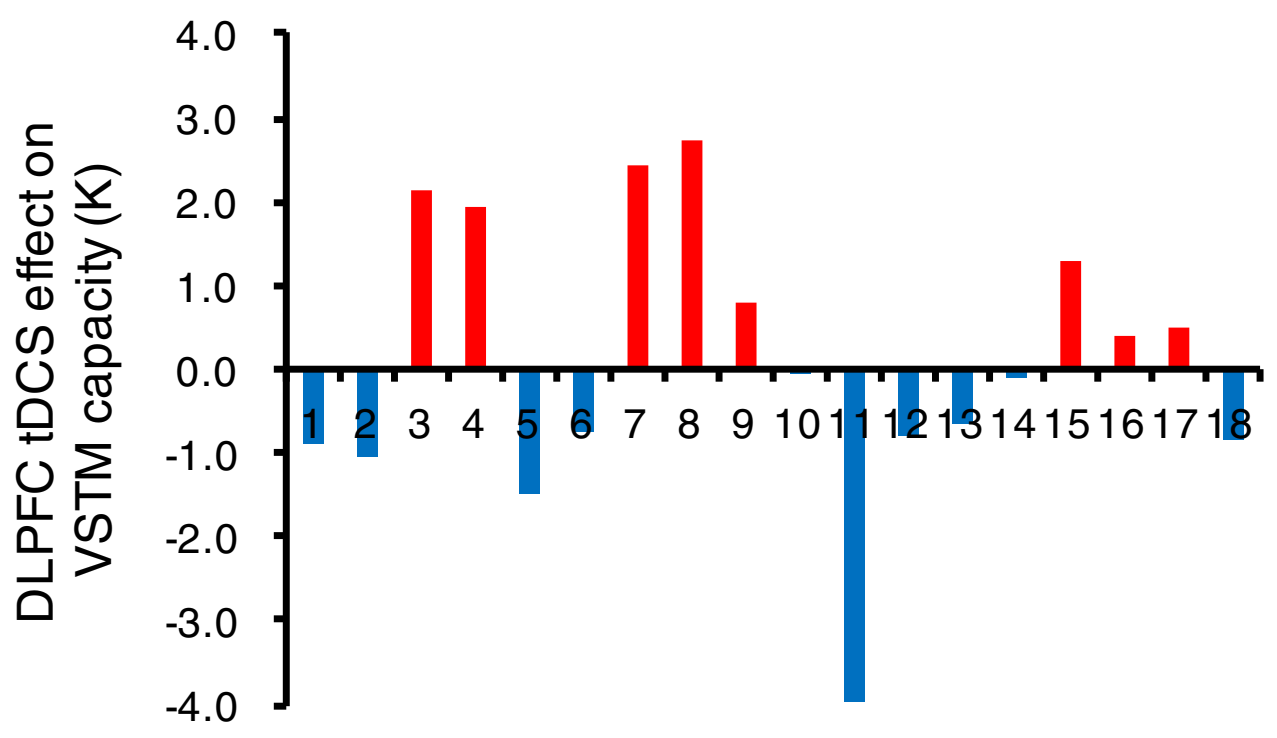

Figure 4. $\boldsymbol{a}, \boldsymbol{b}$, The individual tDCS effects on VSTM capacity with the PPC ( $\boldsymbol{a})$ and DLPFC (b) stimulation at set size 6 . The red bars represent tDCS increasing VSTM capacity relative to sham, and the blue bars represent tDCS decreasing VSTM capacity relative to sham.

2017; Yu and Shim, 2017). However, our tDCS results showed that stimulating the PPC could only enhance the capacity of VSTM, but it was not sufficient to increase the precision of VSTM. The null effect of PPC stimulation on the precision parameter is consistent with the observation that brain regions other than the PPC, such as occipital and frontal cortices, also encode sensory representations during the maintenance period of VSTM (Harrison and Tong, 2009; Serences et al., 2009; Ester et al., 2013, 2015; Sprague et al., 2014, 2016; Bettencourt and Xu, 2016; Galeano Weber et al., 2017). In contrast to this null PPC stimulation effect, a recent study has shown that stimulating early visual areas using transcranial magnetic stimulation (TMS) could induce changes in VTSM precision (Rademaker et al., 2017). Differential effects of stimulating the PPC and visual cortex found across our present study and the recent TMS study suggest that these two brain areas may serve differential VSTM functions, as has actively debated in the WM literature (Ester et al., 2015, 2016; but see Bettencourt and Xu, 2016; Leavitt et al., 2017; Xu, 2017, 2018; Gayet et al., 2018; Scimeca et al., 2018). Specifically, the PPC may have a more dominant role in providing the storage for discrete items in VSTM, but the occipital cortex is relatively better at preserving the quality of sensory representations necessary for behavioral tasks where subjects have to remember and recall fine-grained details of visual stimuli.

Since tDCS allows the flow of tonic direct current to pass through the cortical tissue, the anodal stimulation should depolarize resting membrane potentials of neurons within the PPC, as shown in the current density distribution model in Figure $1 c$. Accordingly, this depolarization should increase the neuronal excitability within this cortical region via voltage-dependent ion channels (Nitsche and Paulus, 2000, 2001; Nitsche et al., 2003; for review, see Polanía et al., 2018). Because we stimulated the PPC over $15 \mathrm{~min}$, this stimulation protocol should also lead to changes in synaptic plasticity regulated by NMDA glutamatergic and 
GABAergic receptors, and this could produce a long-lasting neuronal excitability effect over hours (Nitsche et al., 2003; Stagg et al., 2009; for review, see Polanía et al., 2018). These neuronal changes could, in turn, improve the excitability of the PPC during the VSTM task, hence increasing the maximum number of objects that can be maintained in VSTM. Future studies could use similar behavioral and modeling approaches in combination with neurobiological assessments (e.g., fMRI and EEG) to test the hypothesis we raised here.

In conclusion, we found that anodal tDCS over PPC selectively improved VSTM capacity without changes in VSTM precision or SM function. These results provide causal evidence suggesting that the PPC has a more dominant role in supporting the storage capacity of VSTM rather than maintaining the quality of sensory representations. Furthermore, tDCS could be used as a promising noninvasive method to enhance VSTM function.

\section{References}

Alloway TP, Alloway RG (2010) Investigating the predictive roles of working memory and IQ in academic attainment. J Exp Child Psychol 106:2029. CrossRef Medline

Andrews SC, Hoy KE, Enticott PG, Daskalakis ZJ, Fitzgerald PB (2011) Improving working memory: the effect of combining cognitive activity and anodal transcranial direct current stimulation to the left dorsolateral prefrontal cortex. Brain Stimul 4:84-89. CrossRef Medline

Baddeley AD, Hitch G (1974) Working memory. Psychol Learn Motiv 8:4789. CrossRef

Bays PM, Husain M (2008) Dynamic shifts of limited working memory resources in human vision. Science 321:851-854. CrossRef Medline

Bettencourt KC, Xu Y (2016) Decoding the content of visual short-term memory under distraction in occipital and parietal areas. Nat Neurosci 19:150-157. CrossRef Medline

Brainard DH (1997) The psychophysics toolbox. Spat Vis 10:433-436. CrossRef Medline

Collins DL, Neelin P, Peters TM, Evans AC (1994) Automatic 3d intersubject registration of mr volumetric data in standardized talairach space. J Comput Assist Tomogr 18:192-205. CrossRef Medline

Colom R, Abad FJ, Quiroga MÁ, Shih PC, Flores-Mendoza C (2008) Working memory and intelligence are highly related constructs, but why? Intelligence 36:584-606. CrossRef

Conway ARA, Cowan N, Bunting MF, Therriault DJ, Minkoff SRB (2002) A latent variable analysis of working memory capacity, short-term memory capacity, processing speed, and general fluid intelligence. Intelligence 30 : 163-183. CrossRef

Cowan N (2001) Metatheory of storage capacity limits. Behav Brain Sci 24: $154-176$.

Elmer S, Burkard M, Renz B, Meyer M, Jancke L (2009) Direct current induced short-term modulation of the left dorsolateral prefrontal cortex while learning auditory presented nouns. Behav Brain Funct 5:29. CrossRef Medline

Ester EF, Anderson DE, Serences JT, Awh E (2013) A neural measure of precision in visual working memory. J Cogn Neurosci 25:754-761. CrossRef Medline

Ester EF, Sprague TC, Serences JT (2015) Parietal and frontal cortex encode stimulus-specific mnemonic representations during visual working memory. Neuron 87:893-905. CrossRef Medline

Ester EF, Rademaker RL, Sprague TC (2016) How do visual and parietal cortex contribute to visual short-term memory? eNeuro 3:ENEURO.004116.2016. CrossRef Medline

Faul F, Erdfelder E, Buchner A, Lang AG (2009) Statistical power analyses using $\mathrm{G}^{*}$ power 3.1: tests for correlation and regression analyses. Behav Res Methods 41:1149-1160. CrossRef Medline

Fregni F, Boggio PS, Nitsche M, Bermpohl F, Antal A, Feredoes E, Marcolin MA, Rigonatti SP, Silva MT, Paulus W, Pascual-Leone A (2005) Anodal transcranial direct current stimulation of prefrontal cortex enhances working memory. Exp Brain Res 166:23-30. CrossRef Medline

Fukuda K, Kang MS, Woodman G (2015a) Electrophysiology reveals distinct neural mechanisms for lateralized and spatially global visual working memory representations. J Vis 15(12):1114. CrossRef

Fukuda K, Mance I, Vogel EK (2015b) Power modulation and event-related slow wave provide dissociable correlates of visual working memory. J Neurosci 35:14009-14016. CrossRef Medline

Galeano Weber EM, Peters B, Hahn T, Bledowski C, Fiebach CJ (2016) Superior intraparietal sulcus controls the variability of visual working memory precision. J Neurosci 36:5623-5635. CrossRef Medline

Galeano Weber EM, Hahn T, Hilger K, Fiebach CJ (2017) Distributed patterns of occipito-parietal functional connectivity predict the precision of visual working memory. Neuroimage 146:404-418. CrossRef Medline

Gayet S, Paffen CLE, Van der Stigchel S (2018) Visual working memory storage recruits sensory processing areas. Trends Cogn Sci 22:189-190. CrossRef Medline

Gazzaley A, Cooney JW, Rissman J, D’Esposito M (2005) Top-down suppression deficit underlies working memory impairment in normal aging. Nat Neurosci 8:1298-1300. CrossRef Medline

Habekost T, Rostrup E (2007) Visual attention capacity after right hemisphere lesions. Neuropsychologia 45:1474-1488. CrossRef Medline

Harrison SA, Tong F (2009) Decoding reveals the contents of visual working memory in early visual areas. Nature 458:632-635. CrossRef Medline

Haueisen J, Ramon C, Eiselt M, Brauer H, Nowak H (1997) Influence of tissue resistivities on neuromagnetic fields and electric potentials studied with a finite element model of the head. IEEE Trans Biomed Eng 44:727735. CrossRef Medline

Heinen K, Sagliano L, Candini M, Husain M, Cappelletti M, Zokaei N (2016) Cathodal transcranial direct current stimulation over posterior parietal cortex enhances distinct aspects of visual working memory. Neuropsychologia 87:35-42. CrossRef Medline

Hsu TY, Tseng P, Liang WK, Cheng SK, Juan CH (2014) Transcranial direct current stimulation over right posterior parietal cortex changes prestimulus alpha oscillation in visual short-term memory task. Neuroimage 98 : 306-313. CrossRef Medline

Jo JM, Kim YH, Ko MH, Ohn SH, Joen B, Lee KH (2009) Enhancing the working memory of stroke patients using tDCS. Am J Phys Med Rehabil 88:404-409. CrossRef Medline

Jung YJ, Kim JH, Im CH (2013) COMETS: a MATLAB toolbox for simulating local electric fields generated by transcranial direct current stimulation (tDCS). Biomed Eng Lett 3:39-46. CrossRef

Kim JH, Kim DW, Chang WH, Kim YH, Kim K, Im CH (2014) Inconsistent outcomes of transcranial direct current stimulation may originate from anatomical differences among individuals: electric field simulation using individual MRI data. Neurosci Lett 564:6-10. CrossRef Medline

Leavitt ML, Mendoza-Halliday D, Martinez-Trujillo JC (2017) Sustained activity encoding working memories: not fully distributed. Trends Neurosci 40:328-346. CrossRef Medline

Lee J, Park S (2005) Working memory impairments in schizophrenia: a meta-analysis. J Abnorm Psychol 114:599-611. CrossRef Medline

Li S, Cai Y, Liu J, Li D, Feng Z, Chen C, Xue G (2017) Dissociated roles of the parietal and frontal cortices in the scope and control of attention during visual working memory. Neuroimage 149:210-219. CrossRef Medline

Loftus AM, Nicholls ME (2012) Testing the activation-orientation account of spatial attentional asymmetries using transcranial direct current stimulation. Neuropsychologia 50:2573-2576. CrossRef Medline

Luck SJ, Vogel EK (1997) The capacity of visual working memory for features and conjunctions. Nature 390:279-281. CrossRef Medline

McCarthy G, Puce A, Constable RT, Krystal JH, Gore JC, Goldman-Rakic P (1996) Activation of human prefrontal cortex during spatial and nonspatial working memory tasks measured by functional MRI. Cereb Cortex 6:600-611. CrossRef Medline

McCollough AW, Machizawa MG, Vogel EK (2007) Electrophysiological measures of maintaining representations in visual working memory. Cortex 43:77-94. CrossRef Medline

Nitsche MA, Paulus W (2000) Excitability changes induced in the human motor cortex by weak transcranial direct current stimulation. J Physiol 527:633-639. CrossRef Medline

Nitsche MA, Paulus W (2001) Sustained excitability elevations induced by transcranial DC motor cortex stimulation in humans. Neurology 57: 1899-1901. CrossRef Medline

Nitsche MA, Fricke K, Henschke U, Schlitterlau A, Liebetanz D, Lang N, Henning S, Tergau F, Paulus W (2003) Pharmacological modulation of cortical excitability shifts induced by transcranial direct current stimulation in humans. J Physiol 553:293-301. CrossRef Medline

Ohn SH, Park CI, Yoo WK, Ko MH, Choi KP, Kim GM, Lee YT, Kim YH (2008) Time-dependent effect of transcranial direct current stimulation 
on the enhancement of working memory. Neuroreport 19:43-47. CrossRef Medline

Owen AM, McMillan KM, Laird AR, Bullmore E (2005) N-back working memory paradigm: a meta-analysis of normative functional neuroimaging studies. Hum Brain Mapp 25:46-59. CrossRef Medline

Pelli DG (1997) The VideoToolbox software for visual psychophysics: transforming numbers into movies. Spat Vis 10:437-442. CrossRef Medline

Polanía R, Nitsche MA, RuffCC (2018) Studying and modifying brain function with non-invasive brain stimulation. Nat Neurosci 21:174-187. CrossRef Medline

Rademaker RL, van de Ven VG, Tong F, Sack AT (2017) The impact of early visual cortex transcranial magnetic stimulation on visual working memory precision and guess rate. PLoS One 12:e0175230. CrossRef Medline

Ramaraju S, Roula MA, McCarthy PW (2018) Modelling the effect of electrode displacement on transcranial direct current stimulation (tDCS). J Neural Eng 15:016019. CrossRef Medline

Reinhart RM, Woodman GF (2014) Causal control of medial-frontal cortex governs electrophysiological and behavioral indices of performance monitoring and learning. J Neurosci 34:4214-4227. CrossRef Medline

Reinhart RM, Woodman GF (2015) Enhancing long-term memory with stimulation tunes visual attention in one trial. Proc Natl Acad Sci U S A 112:625-630. CrossRef Medline

Reinhart RM, Xiao W, McClenahan LJ, Woodman GF (2016) Electrical stimulation of visual cortex can immediately improve spatial vision. Curr Biol 26:1867-1872. CrossRef Medline

Rose EJ, Ebmeier KP (2006) Pattern of impaired working memory during major depression. J Affect Disord 90:149-161. CrossRef Medline

Roy LB, Sparing R, Fink GR, Hesse MD (2015) Modulation of attention functions by anodal tDCS on right PPC. Neuropsychologia 74:96-107. CrossRef Medline

Scimeca JM, Kiyonaga A, D’Esposito M (2018) Reaffirming the sensory recruitment account of working memory. Trends Cogn Sci 22:190-192. CrossRef Medline

Serences JT, Ester EF, Vogel EK, Awh E (2009) Stimulus-specific delay activity in human primary visual cortex. Psychol Sci 20:207-214. CrossRef Medline

Smith EE, Jonides J, Koeppe RA (1996) Dissociating verbal and spatial working memory using PET. Cereb Cortex 6:11-20. CrossRef Medline

Sparing R, Thimm M, Hesse MD, Küst J, Karbe H, Fink GR (2009) Bidirectional alterations of interhemispheric parietal balance by non-invasive cortical stimulation. Brain 132:3011-3020. CrossRef Medline

Sperling G (1960) The information available in brief visual presentations. Psychol Monogr Gen Appl 74:1-29. CrossRef

Sprague TC, Ester EF, Serences JT (2014) Reconstructions of information in visual spatial working memory degrade with memory load. Curr Biol 24:2174-2180. CrossRef Medline

Sprague TC, Ester EF, Serences JT (2016) Restoring latent visual working memory representations in human cortex. Neuron 91:694-707. CrossRef Medline

Stagg CJ, Best JG, Stephenson MC, O’Shea J, Wylezinska M, Kincses ZT, Morris PG, Matthews PM, Johansen-Berg H (2009) Polarity-sensitive modulation of cortical neurotransmitters by transcranial stimulation. J Neurosci 29:5202-5206. CrossRef Medline

Suchow J, Brady T (2013) Modeling visual working memory with the MemToolbox. J Vis 13(10):9, 1-8. CrossRef Medline

Swanson HL (1994) Short-term memory and working memory: do both contribute to our understanding of academic achievement in children and adults with learning disabilities? J Learn Disabil 27:34-50. CrossRef Medline

Todd JJ, Marois R (2004) Capacity limit of visual short-term memory in human posterior parietal cortex. Nature 428:751-754. CrossRef Medline

Todd JJ, Marois R (2005) Posterior parietal cortex activity predicts individual differences in visual short-term memory capacity. Cogn Affect Behav Neurosci 5:144-155. CrossRef Medline

Tseng P, Hsu TY, Chang CF, Tzeng OJ, Hung DL, Muggleton NG, Walsh V, Liang WK, Cheng SK, Juan CH (2012) Unleashing potential: transcranial direct current stimulation over the right posterior parietal cortex improves change detection in low-performing individuals. J Neurosci 32:10554-10561. CrossRef Medline

van der Ham IJ, Raemaekers M, van Wezel RJ, Oleksiak A, Postma A (2009) Categorical and coordinate spatial relations in working memory: an fMRI study. Brain Res 1297:70-79. CrossRef Medline

Vogel EK, Machizawa MG (2004) Neural activity predicts individual differences in visual working memory capacity. Nature 428:748-751. CrossRef Medline

Vogel JJ, Bowers CA, Vogel DS (2003) Cerebral lateralization of spatial abilities: a meta-analysis. Brain Cogn 52:197-204. CrossRef Medline

Wager TD, Smith EE (2003) Neuroimaging studies of working memory. Cogn Affect Behav Neurosci 3:255-274. CrossRef Medline

Walter H, Bretschneider V, Grön G, Zurowski B, Wunderlich AP, Tomczak R, Spitzer M (2003) Evidence for quantitative domain dominance for verbal and spatial working memory in frontal and parietal cortex. Cortex 39:897-911. CrossRef Medline

Xu Y (2017) Reevaluating the sensory account of visual working memory storage. Trends Cogn Sci 21:794-815. CrossRef Medline

$\mathrm{Xu}$ Y (2018) Sensory cortex is nonessential in working memory storage. Trends Cogn Sci 22:192-193. CrossRef Medline

Xu Y, Chun MM (2006) Dissociable neural mechanisms supporting visual short-term memory for objects. Nature 440:91-95. CrossRef Medline

Xue G, Juan CH, Chang CF, Lu ZL, Dong Q (2012) Lateral prefrontal cortex contributes to maladaptive decisions. Proc Natl Acad Sci U S A 109:44014406. CrossRef Medline

Yu Q, Shim WM (2017) Occipital, parietal, and frontal cortices selectively maintain task-relevant features of multi-feature objects in visual working memory. Neuroimage 157:97-107. CrossRef Medline

Zaehle T, Sandmann P, Thorne JD, Jäncke L, Herrmann CS (2011) Transcranial direct current stimulation of the prefrontal cortex modulates working memory performance: combined behavioural and electrophysiological evidence. BMC Neurosci 12:2. CrossRef Medline

Zhang W, Luck SJ (2008) Discrete fixed-resolution representations in visual working memory. Nature 453:233-235. CrossRef Medline

Zhang W, Luck SJ (2011) The number and quality of representations in working memory. Psychol Sci 22:1434-1441. CrossRef Medline 